\section{Evolução Materno-Fetal de Gestantes Diabéticas Seguidas no HC-FMRP-USP no Periodo de 1992-1999}

\section{RESUMO}

Este estudo teve por objetivo avaliar a freqüência de complicações materno-fetais, tipo de parto e controle metabólico das gestantes diabéticas atendidas no HCFMRP-USP, entre 1992 e 1999. Foram estudadas 261 pacientes, das quais 44 (16,3\%) tinham diabetes mellitus tipo 1 (DM1), 82 (30,5\%) diabetes tipo 2 (DM2) e 143 (53,2\%) diabetes gestacional (DMG). Observou-se uma freqüência elevada de obesidade previamente à gestação nas pacientes com DMG $(47,6 \%)$ e DM2 $(65,9 \%)$ e também de HAS nesse último grupo (46,3\%). Apesar do início tardio, houve no decorrer do seguimento melhora do controle metabólico do DM nos 3 grupos, observada através da redução dos níveis glicêmicos (DM1 às 10h: $197 \pm 40$ vs. $128 \pm 39 \mathrm{mg} / \mathrm{dl}, \mathrm{p}=0,003$; $\mathrm{DM} 2$ às $7 \mathrm{~h}: 147 \pm 53$ vs. $102 \pm 19 \mathrm{mg} / \mathrm{dl}, \mathrm{p}=0,001 ; 14 \mathrm{~h}: 164 \pm 53$ vs. $121 \pm 28 \mathrm{mg} / \mathrm{dl}, \mathrm{p}=0,01 ;$ e $20 \mathrm{~h}$ : $201 \pm 55$ vs. $147 \pm 43 \mathrm{mg} / \mathrm{dl}, \mathrm{p}=0,01$; DMG às $7 \mathrm{~h}$ : $100 \pm 34$ vs. $89 \pm 20 \mathrm{mg} / \mathrm{dl}$, $\mathrm{p}=0,003$; $10 \mathrm{~h}: 144 \pm 49$ vs. $122 \pm 29 \mathrm{mg} / \mathrm{dl}, \mathrm{p}=0,03$ e $14 \mathrm{~h}: 126 \pm 38$ vs. $112 \pm 27 \mathrm{mg} / \mathrm{dl}, \mathrm{p}=0,001$ ) e da HbAl (DM1: 11, $\pm 2,9$ vs. 5,7 $\pm 1,8 \%, \mathrm{p}=0,02$ ). As complicações maternas mais incidentes foram hipoglicemias, infecções do trato urinário (ITU), vulvovaginites, hipertensão arterial sistêmica (HAS) e doença hipertensiva específica da gravidez (DHEG). Foi significativamente maior a freqüência de hipoglicemias (29,5\%, $\mathrm{p}<0,0001)$, de ITU (29,5\%, $\mathrm{p}=0,02)$ e de abortos $(11,4 \%, \mathrm{p}=0,003)$ nas pacientes com DM1 que nos outros grupos. Não houve nenhum óbito materno. Nos 3 grupos, o parto cesária foi o mais utilizado (DM1: 74,3\%; DM2: 79,5\%; DMG: 60,5\%). Hipoglicemias, prematuridade, icterícia e macrossomia foram as complicações fetais de maior incidência. Foram complicações significativamente mais freqüentes nos recém-nascidos de gestantes com DM1: prematuridade $(53,7 \%, \mathrm{p}<0,0001)$, natimortalidade $(14,6 \%, p<0,0001)$ e síndrome do desconforto respiratório do recém-nascido (13,9\%, $\mathrm{p}=0,003)$. Embora tenha havido melhora do controle metabólico nos grupos estudados, não foi atingida uma completa normalização dos níveis sangüíneos de glicose e hemoglobina glicada, o que provavelmente contribuiu para as taxas de complicações materno-fetais verificadas nas nossas pacientes. (Arq Bras Endocrinol Metab $2001 ; 45 / 5: 467-474$ )

Unitermos: Diabetes melliłus; Gravidez; Complicações; Controle glicêmico.

\section{artigo original}

\author{
Renan M. Montenegro Jv \\ Glóvia M.F.G. Paccola \\ Cláudia M. Faria \\ Ana P.M. Sales \\ Ana P.D.R. Montenegro \\ Salim M. Jorge \\ Geraldo Duarte \\ Milton C. Foss
}

Divisão de Endocrinologia e Metabologia do Departamento de Clínica Médica; Departamento de Ginecologia e Obstetrícia e Departamento de Pediatria; Faculdade de Medicina de Ribeirão Preto-USP, Ribeirão Preto, SP.

\begin{abstract}
The objective of the present study was to evaluate the frequency of maternal and fetal complications, type of delivery and metabolic control of diabetic pregnant women followed at HCFMRP-USP, between 1992 and 1999. Outcome data were obtained on 261 patients where 44 (16.3\%) had type 1 (DM1), 82 (30.5\%) had type 2 (DM2) and 143 (53.2\%) had gestational diabetes mellitus (GDM). The occurrence of obesity prior to gestation was elevated in patients with GDM (47.6\%) and DM2 (65.9\%). Hypertension was also frequent in the latter group (46.3\%). In spite of the late beginning of the prenatal care in all three groups, there was metabolic control improvement, observed through reduction of glycemias (DM1: 10h: 197 \pm 40 vs. $128 \pm 39 \mathrm{mg} / \mathrm{dl}, \mathrm{p}=0.003 ; \mathrm{DM} 2: 7 \mathrm{~h}: 147 \pm 53$ vs. $102 \pm 19 \mathrm{mg} / \mathrm{dl}, \mathrm{p}=0.001 ; 14 \mathrm{~h}$ : $164 \pm 53$ vs. $121 \pm 28 \mathrm{mg} / \mathrm{dll}, \mathrm{p}=0.01 ; 20 \mathrm{~h}: 201 \pm 55$ vs. $147 \pm 43 \mathrm{mg} / \mathrm{dll}, \mathrm{p}=0.01$;
\end{abstract}

Recebido em 09/10/00 Revisado em 04/04/01 Aceito em 13/07/01 
DMG: 7h: $100 \pm 34$ vs. $89 \pm 20 \mathrm{mg} / \mathrm{dl}, \mathrm{p}=0.003 ; 10 \mathrm{~h}: 144 \pm 49$ vs. $122 \pm 29 \mathrm{mg} / \mathrm{dl}, \mathrm{p}=0.03$; $14 \mathrm{~h}: 126 \pm 38$ vs. $112 \pm 27 \mathrm{mg} / \mathrm{dl}$, $\mathrm{p}=0.001$ ), and $\mathrm{HbA} 1$ levels (DM1: $11.1 \pm 2.9$ vs. $5.7 \pm 1.8 \%$, $\mathrm{p}=0.02)$. Hypoglycemia, urinary tract infections, vulvovaginites, hypertension and pre-eclampsia/ eclampsia were the most incident maternal complications. Hypoglycemia (29.5\%, $\mathrm{p}<0.0001)$, urinary tract infections (29.5\%, $p=0.02)$, and abortions ( $11.4 \%, p=0.003$ ) were significantly more common in DMl patients (11.4\%) than in other groups. No maternal death was observed. Cesarea section was the mode of delivery predominantly used in all groups. Hypoglycemia, prematurity, jaundice and macrossomia were the most incident fetal complications. Prematurity (53.7\%, $\mathrm{p}<0.0001)$, natimortality (14.6\%, $\mathrm{p}<0.0001)$, and respiratory distress syndrome $(13.9 \%, p=0.003)$ were more frequent in newborns of DM1 patients. Although some degree of metabolic control improvement has been observed in all groups, a complete normalization of glucose and glycated hemoglobin levels has not been achieved, and probably contributed for the maternal and fetal complications rates observed. (Arq Bras Endocrinol Metab 2001;45/5:467-474)

Keywords: Diabetes mellitus; Pregnancy; Complications; Glycemic control.

$\mathrm{N}$ AS ÚLTIMAS DÉCADAS, tem havido uma evolução importante no manejo de gestações em pacientes diabéticas, com conseqüente redução da morbidade e mortalidade materno-fetal. Esses resultados são provavelmente devidos à adoção de medidas para maior adesão ao tratamento, controle metabólico mais rígido, atenção mais efetiva à gestante nas últimas semanas da gravidez e implementação de cuidados peri e neonatais intensivos. Apesar desse progresso, complicações maternas como hipoglicemia, cetoacidose, hipertensão arterial sistêmica (HAS), doença hipertensiva específica da gravidez (DHEG), abortos e infecções; e fetais como macrossomia, malformações, prematuridade, hipoglicemia, síndrome do desconforto respiratório do recém-nascido (SDRRN), hipocalcemia, hiperbilirrubinemia e natimortalidade ainda constituem um problema significativo. A gestação em mulheres com DM, tanto as com diagnóstico anterior à gestação quanto aquelas com intolerância à glicose detectada pela primeira vez durante a gravidez, é ainda uma condição reconhecidamente associada a uma maior freqüência dessas complicações quando comparada a mulheres sem DM (1-5).

A morbi-mortalidade materna e fetal aumentadas em gestantes com DM têm sido associadas a fatores como hiperglicemia, hiperinsulinemia, presença de co-morbidades e complicações agudas e crônicas do
DM. A indefinição em relação a alguns aspectos do controle glicêmico parece contribuir consideravelmente para isso. Enquanto o benefício de um controle metabólico materno adequado está bem estabelecido, o nível de manutenção da glicemia necessário para reduzir a incidência de complicações maternas e neonatais permanece indeterminado.

No presente estudo objetivamos avaliar as freqüências de diabetes mellitus tipo 1 (DMl), tipo 2 (DM2) e diabetes mellitus gestacional (DMG) e as características clínicas de cada grupo, com ênfase em relação ao controle metabólico e a ocorrência de complicações maternas e fetais das gestantes seguidas no Hospital das Clínicas da Faculdade de Medicina de Ribeirão Preto - USP entre 1992 e 1999.

\section{MATERIAL E MÉTODOS}

Avaliamos 261 gestantes com DM atendidas no Ambulatório de Pré-Natal Patológico do Hospital das Clínicas da Faculdade de Medicina de Ribeirão Preto - USP, no período de 1992 a 1999. Todas as informações foram obtidas a partir de dados do protocolo de seguimento endocrinológico da gestante, preenchida a cada consulta com informações dirigidas ao DM e co-morbidades e confirmados com os dados do prontuário médico materno e do recémnascido. As pacientes foram classificadas em DMl ou DM2, de acordo com o diagnóstico prévio estabelecido clinicamente (presença de obesidade, história familiar de DM, episódios prévios de cetoacidose e terapêutica com insulina), e DMG, caso a intolerância à glicose tivesse sido detectada pela primeira vez durante a gestação $(6,7)$. O diagnóstico de DMG foi baseado nos critérios do National Diabetes Data Group, 1991 (7). As pacientes foram avaliadas quanto a idade, raça, período gestacional de início do seguimento, paridade, presença de complicações do DM (nefro, neuro, retino e macroangiopatia), comorbidades (obesidade, hipertensão arterial sistêmica, dislipidemia prévia) e história familiar de diabetes. O grupo com DMG foi ainda avaliado quanto a ocorrência de DMG em gestações anteriores. As complicações crônicas de DM e co-morbidades foram avaliadas na primeira consulta e a cada trimestre da gestação, através de dosagem sérica de nitrogênio uréico, creatinina, sódio, potássio, colesterol total, HDLcolesterol, triglicerídeos; da determinação da proteinúria de 24hs; da realização de urina rotina (urina I) e urocultura; da realização de fundoscopia (por oftalmologista) e de um eletrocardiograma. A ocorrência de neuropatia foi avaliada pelo endocrinolo- 
gista, utilizando-se critérios clínicos (anamnese e exame físico).

No início do seguimento todas as pacientes recebiam orientação dietética adequada para o seu perfil metabólico e ponderal, realizada pela nutricionista da equipe de trabalho, sendo introduzido ou modificado o esquema de insulinoterapia caso não fosse obtido um controle satisfatório. Os antidiabéticos orais foram suspensos na $\mathrm{l}^{\text {a }}$ consulta nas pacientes que se encontravam em uso inadvertido. A insulinoterapia, quando indicada, foi iniciada com uma insulina de ação intermediária (NPH ou lenta) em dose única matinal $(0,3 \mathrm{U} / \mathrm{kg} /$ dia $)$. Para adequação do esquema foi levado em conta o controle glicêmico e ganho de peso. De acordo com esses parâmetros, foram feitos os ajustes, sendo utilizadas doses múltiplas de insulina de ação intermediária, associadas ou não às de ação rápida. O aumento da dose de insulina era de aproximadamente $20 \%$ da dose anterior, sempre se utilizando como critérios o ganho ponderal, o perfil glicêmico e a resposta ao último aumento de dose. O controle glicêmico foi avaliado pela mediana das glicemias de jejum e pós-prandiais determinadas, em sangue venoso, a cada retorno ao ambulatório e durante as internações e das hemoglobinas glicadas solicitadas trimestralmente. Eram colhidas amostras para glicemia às $7 \mathrm{hs}$ (em jejum) e às $10,14,20 \mathrm{e} 2 \mathrm{hs}$. Os parâmetros considerados de bom controle foram: glicemia de jejum < $<105 \mathrm{mg} / \mathrm{dl}$; pré-prandial e pós-prandial $<120 \mathrm{mg} / \mathrm{dl}$; noturna $(2 \mathrm{~h})$ entre 60 e $120 \mathrm{mg} / \mathrm{dl}(7)$. As pacientes tinham retornos a cada 1 a 2 semanas, sendo internadas se apresentassem um controle metabólico ruim. Após a 32a semana da gestação os retornos eram semanais. Os partos foram acompanhados por pediatra da Unidade de Neonatologia. Os recém-nascidos $(\mathrm{RN})$ foram examinados imediatamente após o nascimento com especial atenção para as anormalidades relacionadas ao DM.

Foram analisadas as seguintes complicações maternas: hipoglicemia, cetoacidose, HAS (de início na gravidez), DHEG, polidrâmnio, oligoâmnio, aborto, óbito materno, trabalho de parto prematuro, amniorrexe prematura, hiperêmese gravídica, hemorragia transvaginal, infecção do trato urinário (ITU), vulvovaginite, e outras infecções.

As complicações fetais avaliadas foram: natimortalidade, prematuridade, macrossomia, malformações, policitemia, hipoglicemia, icterícia, SDRRN, sepse, síndrome hemorrágica do recém-nascido, incompatibilidade $\mathrm{ABO}$, transtorno transitório do recém-nascido (TTRN) e a ocorrência de apresentação pélvica, tocotraumatismo e gemelaridade.
A glicose plasmática foi dosada em duplicata, pelo método enzimático da glicose-oxidase, através de analisador de glicose (Beckman Instruments, Furlleton, $\mathrm{CA}$, USA). A hemoglobina glicada $\left(\mathrm{HbA}_{1}\right)$ foi determinada por cromatografia de afinidade (Pierce, Rockford, IL, USA) cujos valores de referência são 3,8-6,0\%.

As medianas das glicemias de cada horário (7, $10,14,20$ e $2 \mathrm{~h})$ e hemoglobina glicada foram comparados entre os três períodos (trimestres) gestacionais nos grupos DM1 e DM2 e entre os dois últimos períodos no grupo DMG, sendo analisados respectivamente através da análise de variância de Friedman e do teste de Wilcoxon, ambos associados ao teste para comparações múltiplas de Dunn. Para a análise das freqüências das diversas características e complicações dos 3 grupos foi utilizado o teste do Qui-quadrado. O nível de significância adotado foi de $5 \%(\mathrm{p}<0,05)$. Para isso foi utilizado o programa Graphpad Prism, versão 3.0.

\section{RESULTADOS}

Das 261 gestantes estudadas, $44(16,3 \%)$ tinham DMl, $82(30,5 \%)$ DM2 e $143(53,2 \%)$ DMG. As características, co-morbidades e complicações existentes previamente à gestação das pacientes dos 3 grupos estão descritas na tabela 1. As gestantes com DM2 apresentaram uma prevalência elevada de HAS e obesidade prévia. Essa última mostrou-se também freqüente nas pacientes com DMG. Os 3 grupos tiveram um início tardio do seguimento no ambulatório especializado, com menos de $50 \%$ das pacientes o fazendo no $\mathrm{l}^{\circ}$. trimestre (tabela $\mathrm{l}$ ).

As medianas das glicemias e hemoglobina glicada de cada grupo são apresentadas na tabela 2. Foi observada uma melhora progressiva do controle metabólico nos 3 grupos no decorrer do seguimento. Evidenciou-se uma redução dos níveis glicêmicos entre o $1^{\circ}$. e o $3^{\circ}$. trimestre nas gestantes com DMl e DM2 e entre o $2^{\circ}$. e $3^{\circ}$. o trimestre nas pacientes que apresentaram DMG, estatisticamente significativo às $10 \mathrm{hs}$ $(\mathrm{p}=0,003)$ no primeiro grupo $(\mathrm{DMl})$, às 7 $(\mathrm{p}=0,001), 14(\mathrm{p}=0,0 \mathrm{l})$ e $20 \mathrm{hs}(\mathrm{p}=0,0 \mathrm{l})$ no segundo $(\mathrm{DM} 2)$ e às $7(\mathrm{p}=0,003), 10(\mathrm{p}=0,03)$ e 14 hs $(\mathrm{p}=0,001)$ no último (DMG). Houve redução da $\mathrm{HbA}_{1}$ nos 3 grupos com o decorrer da gestação, porém estatisticamente significativa apenas nas pacientes com DMl $(\mathrm{p}=0,02)$.

As medianas das glicemias e hemoglobina glicada de cada grupo são apresentadas na tabela 2. Foi observada uma melhora progressiva do controle metabólico nos 3 grupos no decorrer do seguimento. Evidenciouse uma redução dos níveis glicêmicos entre o $1^{\circ}$. e o $3^{\circ}$. 
Tabela 1. Características prévias à gestação e início do seguimento das pacientes avaliadas com DM1, DM2 e DMG.

\begin{tabular}{|c|c|c|c|}
\hline & DM1 $(n=44)$ & $\mathrm{DM} 2(\mathrm{n}=82)$ & $D M G(n=143)$ \\
\hline Idade (anos) & $25 \pm 5,8$ & $33 \pm 5,1$ & $32 \pm 6,0$ \\
\hline Tempo de diagnóstico do DM (anos) & $9,7 \pm 5,8$ & $4,6 \pm 4,1$ & - \\
\hline Raça branca & $77,3 \%(34)$ & $69,5 \%(57)$ & $69,9 \%(100)$ \\
\hline mestiça & $4,5 \%(8)$ & $8,5 \%(7)$ & $10,5 \%(15)$ \\
\hline negra & $18,2 \%(2)$ & $22,0 \%(18)$ & $18,9 \%(27)$ \\
\hline amarela & $0 \%(0)$ & $0 \%(0)$ & $0,7 \%(1)$ \\
\hline Obesidade prévia (IMC $\geq 30 \mathrm{~kg} / \mathrm{m} 2)$ & $6,8 \%(3)$ & $65,9 \%(54)$ & $47,6 \%(68)$ \\
\hline HAS prévia & $6,8 \%(3)$ & $46,3 \%(38)$ & $22,4 \%(32)$ \\
\hline Hiperlipemia prévia & $10,3 \%(4)$ & $9,0 \%(6)$ & $2,1 \%(3)$ \\
\hline Nefropatia prévia & $11,4 \%(5)$ & $1,2 \%(1)$ & - \\
\hline Retinopatia prévia & $13,6 \%(6)$ & $0 \%(0)$ & - \\
\hline Neuropatia prévia & $4,5 \%(2)$ & $0 \%(0)$ & - \\
\hline Macroangiopatia prévia & $0 \%(0)$ & $2,4 \%(2)$ & - \\
\hline DMG prévio & - & $17,1 \%(14)$ & $11,2 \%(16)$ \\
\hline História familiar de DM & $52,3 \%(23)$ & $70,7 \%(58)$ & $55,2 \%(79)$ \\
\hline Multiparidade & $65,9 \%(29)$ & $91,5 \%(75)$ & $8,9 \%(120)$ \\
\hline \multicolumn{4}{|l|}{ Início do seguimento: } \\
\hline $1^{\circ}$ trimestre & $40,9 \%(18)$ & $35,4 \%(29)$ & $0,7 \%(1)$ \\
\hline $2^{\circ}$ trimestre & $34,1 \%(15)$ & $42,7 \%(35)$ & $33,6 \%(48)$ \\
\hline $3^{\circ}$ trimestre & $25,0 \%(11)$ & $21,9 \%(18)$ & $65,7 \%(94)$ \\
\hline
\end{tabular}

(-) Os valores entre parênteses representam os números absolutos de pacientes avaliadas.

trimestre nas gestantes com DMl e DM2 e entre o $2^{\circ}$. e $3^{\circ}$. trimestre nas pacientes que apresentaram DMG, estatisticamente significativo às $10 \mathrm{hs}(\mathrm{p}=0,003)$ no primeiro grupo $(\mathrm{DMl})$ e às $7(\mathrm{p}=0,001), 14(\mathrm{p}=0,01)$ e $20 \mathrm{hs}(\mathrm{p}=0,01)$ no segundo $(\mathrm{DM} 2)$ e às $7(\mathrm{p}=0,003)$, $10(\mathrm{p}=0,03)$ e $14 \mathrm{hs}(\mathrm{p}=0,001)$ no último $(\mathrm{DMG})$.
Houve redução da $\mathrm{HbA}_{1}$ nos 3 grupos com o decorrer da gestação, porém estatisticamente significativa apenas nas pacientes com DMl $(\mathrm{p}=0,02)$.

As complicações maternas estão descritas na tabela 3. As mais incidentes nos 3 grupos estudados foram os episódios de hipoglicemia, as intercorrências

Tabela 2. Controle metabólico (média $\pm D P)$ de pacientes avaliadas com DM1 ( $n=44)$, DM2 $(n=82)$ e $D M G(n=143)$.

\begin{tabular}{|c|c|c|c|c|c|c|}
\hline & \multicolumn{5}{|c|}{ Glicemia (mg/dl) } & \multirow{2}{*}{$\frac{\mathrm{HbA} 1}{(\%)}$} \\
\hline & $7 \mathrm{~h}$ & $10 \mathrm{~h}$ & $14 \mathrm{~h}$ & $20 \mathrm{~h}$ & $2 \mathrm{~h}$ & \\
\hline $\begin{array}{l}\text { DM1 } 1^{\circ} \text { trimestre } \\
(n=18)\end{array}$ & $99 \pm 41$ & $197 \pm 40$ & $197 \pm 64$ & $184 \pm 69$ & $121 \pm 115$ & $11,1 \pm 2,9$ \\
\hline $\begin{array}{l}\text { DM1 } 2^{\circ} \text { trimestre } \\
(n=33)\end{array}$ & $96 \pm 43$ & $162 \pm 31$ & $124 \pm 43$ & $152 \pm 56$ & $157 \pm 66$ & $7,0 \pm 3,0$ \\
\hline $\begin{array}{l}\text { DM1 } 3^{\circ} \text { trimestre } \\
(n=44)\end{array}$ & $88 \pm 44$ & $128 \pm 39$ & $127 \pm 50$ & $140 \pm 46$ & $130 \pm 34$ & $5,7 \pm 1,8$ \\
\hline $\begin{array}{l}\text { DM2 } 1^{\circ} \text { trimestre } \\
(n=29)\end{array}$ & $147 \pm 53$ & $188 \pm 69$ & $164 \pm 53$ & $201 \pm 55$ & $192 \pm 68$ & $7,8 \pm 3,9$ \\
\hline $\begin{array}{l}\text { DM2 } 2^{\circ} \text { trimestre } \\
(n=64)\end{array}$ & $109 \pm 33$ & $152 \pm 42$ & $122 \pm 40$ & $158 \pm 39$ & $169 \pm 37$ & $6,6 \pm 2,7$ \\
\hline $\begin{array}{l}\text { DM2 } 3^{\circ} \text { trimestre } \\
(n=82)\end{array}$ & $102 \pm 19$ & $143 \pm 31$ & $121 \pm 28$ & $147 \pm 43$ & $131 \pm 35$ & $6,0 \pm 2,1$ \\
\hline $\begin{array}{l}\text { DMG } 2^{\circ} \text { trimestre } \\
(n=48)\end{array}$ & $100 \pm 34$ & $144 \pm 49$ & $126 \pm 38$ & $207 \pm 57$ & $174 \pm 48$ & $5,5 \pm 2,8$ \\
\hline $\begin{array}{l}\text { DMG } 3^{\circ} \text { trimestre } \\
(n=142)\end{array}$ & $89 \pm 20$ & $122 \pm 29$ & $112 \pm 27$ & $133 \pm 43$ & $144 \pm 41$ & $5,4 \pm 2,8$ \\
\hline
\end{tabular}

(-) Os valores entre parêntesis representam os números absolutos de pacientes avaliadas. 
Tabela 3. Freqüência (\%) de complicações das gestantes avaliadas com DM1, DM2 e DMG.

\begin{tabular}{|c|c|c|c|}
\hline & $\begin{array}{c}\text { DM1 } \\
(n=44)\end{array}$ & $\begin{array}{c}\text { DM2 } \\
(n=82)\end{array}$ & $\begin{array}{c}\text { DMG } \\
(n=143)\end{array}$ \\
\hline ITU & $29,5(13)^{*}$ & $19,5(16)$ & $11,9(17)$ \\
\hline Hipoglicemia & $29,5(13)^{*}$ & $8,5(7)$ & $5,6(8)$ \\
\hline Vulvovaginite & $13,6(6)$ & $9,7(8)$ & $8,4(12)$ \\
\hline HAS & $13,6(6)$ & $4,9(4)$ & $11,2(16)$ \\
\hline DHEG & $6,8(3)$ & $8,5(7)$ & $9,8(14)$ \\
\hline Aborto & $11,4(5)^{*}$ & $3,7(3)$ & $0,7(1)$ \\
\hline Polidrâmnio & $4,5(2)$ & $0(0)$ & $4,2(6)$ \\
\hline Oligoâmnio & $2,3(1)$ & $4,9(4)$ & $2,1(3)$ \\
\hline Cetoacidose & $4,5(2)$ & $0(0)$ & $0,7(1)$ \\
\hline Amniorrexe prematura & $4,5(2)$ & $1,2(1)$ & $1,4(2)$ \\
\hline Trabalho de parto prematuro & $2,3(1)$ & $1,2(1)$ & $0,7(1)$ \\
\hline Outras infeccões & $2,3(1)$ & $1,2(1)$ & $2,8(4)$ \\
\hline Óbito Materno & $0(0)$ & $0(0)$ & $0(0)$ \\
\hline
\end{tabular}

(-) Os valores entre parêntesis representam os números absolutos de pacientes com as complicações.

* Diferença em relação aos outros 2 grupos $(p<0,05)$.

Tabela 4. Freqüência (\%) de complicações fetais das gestantes avaliadas com DM1, DM2 e DMG.

\begin{tabular}{lcrr}
\hline & $\begin{array}{c}\text { DM1 } \\
(n=41)\end{array}$ & $\begin{array}{c}\text { DM2 } \\
(n=81)\end{array}$ & $\begin{array}{r}\text { DMG } \\
(n=142)\end{array}$ \\
\hline Hipoglicemia & $58,3(21)$ & $62,5(50)$ & $48,6(69)$ \\
Prematuridade & $53,7(22)^{*}$ & $24,7(20)$ & $19,0(27)$ \\
Icterícia & $38,9(14)$ & $27,5(22)$ & $25,4(36)$ \\
Macrossomia & $28,6(11)$ & $22,2(18)$ & $24,6(35)$ \\
Apresentação pélvica & $19,5(8)$ & $11,1(9)$ & $11,3(16)$ \\
Natimortalidade & $14,6(6)^{*}$ & $1,2(1)$ & $0(0)$ \\
SDRRN & $13,9(5)^{*}$ & $1,2(1)$ & $1,4(2)$ \\
Policitemia & $11,1(4)$ & $6,2(5)$ & $2,8(4)$ \\
Malformações & $7,3(3)$ & $11,1(9) * *$ & $1,4(2)$ \\
TTRN & $5,6(2)$ & $6,2(5)$ & $4,2(6)$ \\
Gemelaridade & $5,1(2)^{*}$ & $2,5(2)$ & $0(0)$ \\
Sepse & $2,8(1)^{*}$ & $0(0)$ & $0(0)$ \\
Incompatibilidade ABO & $2,8(1)$ & $1,2(1)$ & $0(0)$ \\
Tocotraumatismo & $2,8(1)$ & $0(0)$ & $0,7(1)$ \\
SHRN & $0(0)$ & $1,2(1)$ & $0(0)$ \\
\hline
\end{tabular}

$\mathrm{n}=$ Número total de fetos avaliados. Difere em relação ao número de gestantes devido aos abortos e gemelares.

(-) Os valores entre parêntesis representam os números absolutos de pacientes com as complicações.

* Diferença em relação aos outros grupos $(p<0,05)$;

** Diferença em relação ao grupo DMG $(p<0,05)$.

infecciosas, principalmente infecções do trato urinário (ITU) e vulvovaginites, e as anormalidades hipertensivas (HAS de início durante a gestação e DHEG). Foi significativamente maior a freqüência de hipoglicemia $(\mathrm{p}<0,0001)$, de ITU $(\mathrm{p}=0,006)$ e de abortos $(\mathrm{p}=0,003)$ nas pacientes com DMl que nos outros grupos. A cetoacidose ocorreu em duas pacientes $(4,5 \%)$ com DMl e uma paciente com DMG. Não houve nenhum caso de óbito materno. O tipo de parto mais comum nos três grupos foi cesária (DMl: 74, $3 \%$, DM2: 79,5\% e DMG: 60,5\%).

As complicações fetais são apresentadas na tabela 4. Hipoglicemia, prematuridade, icterícia e macrossomia foram as de maior incidência nos três grupos. Foram complicações significativamente mais freqüentes nos filhos de diabéticas do tipo 1 prematuridade $(\mathrm{p}<0,0001)$, natimortalidade $(\mathrm{p}<0,0001)$ e SDRRN $(p=0,003)$.

\section{DISCUSSÃO}

Durante a gestação ocorre uma adaptação hormonal, representada por aumento dos níveis de estrógenos, progesterona, cortisol, prolactina e a produção de lactogênio placentário humano. Essas mudanças interferem no equilíbrio metabólico podendo resultar, em mulheres susceptíveis, no desencadeamento de DMG e naquelas previamente diabéticas em uma piora do controle glicêmico.

A importância do bom controle glicêmico durante a gestação em diabéticas está bem determinada há várias décadas. Em relação ao efeito tóxico sobre o feto, foi demonstrado que a hiperglicemia nesse período está associada a uma mortalidade aumentada, além de uma maior freqüência de complicações como macrossomia, hipoglicemia, hiperbilirrubinemia, policitemia, hipocalcemia, hipomagnesemia, cardiomiopatia hipertrófica e SDRRN. Quando a hiperglicemia está presente no início da gestação, durante a fase de organogênese, há um risco aumentado de malformações e abortos espontâneos $(1,8,9)$. As repercussões do mau controle metabólico sobre a gestante são também extremamente negativas. Resulta, dentre outras, em maiores índices de infecções, hipertensão arterial, pré-eclâmpsia, partos cesáreas e pré-termo (10-12).

Observamos, no presente estudo, uma melhora do controle glicêmico nos 3 grupos, no decorrer do período gestacional. Algumas medidas adotadas que provavelmente contribuíram para tal foram: a freqüência dos retornos, na maioria das pacientes de 1 a 2 semanas durante todo o seguimento, e internações nos casos mal controlados, buscando uma monitorização glicêmica razoável, compensando, pelo menos parcialmente, a não adoção por questões sócio-econômicas da monitorização domiciliar diária através de glicemia capilar; a disponibilidade para aquisição gratuita da insulina na rede pública de Ribeirão Preto; a persistência em relação à orientação dietética, a cada retorno e durante a internação; a pronta utilização e ajuste da insulinoterapia nos casos não controlados com a dieta. 
Em relação às complicações maternas pudemos observar, neste estudo, que a maioria delas foi menos incidente que o descrito em outras casuísticas. A hipoglicemia materna tem sido relatada como uma complicação freqüente da gestação de diabéticas, com uma ocorrência de até $72 \%$ em DMl em outras séries $(13,14)$. No presente trabalho, essa complicação ocorreu nos 3 grupos em taxas inferiores a 30\% (tabela 3 ). Isso decorre provavelmente do grau de orientação e cuidado em relação a esse grave efeito adverso da terapêutica do diabetes. Por outro lado, pode sugerir a necessidade de esquema de insulinização ainda mais intensivo, levando-se em conta o controle metabólico atingido e a freqüência de algumas complicações fetais em níveis maiores que de gestantes sem DM.

Mesmo fora do período gestacional, pacientes com DM mal controlado são mais susceptíveis a infecções. Atribui-se a anormalidades na atividade leucocitária e dos linfócitos. Durante a gravidez essa predisposição torna-se maior, devido à deficiência imunológica que essa condição propicia, principalmente em relação à imunidade celular (13). Apesar das infecções do trato urinário e de feridas cirúrgicas pósparto serem reconhecidamente mais freqüentes em diabéticas, a incidência dessas complicações não está bem definida. Stamler e cols. (15) descreveram pelo menos um episódio infeccioso durante período gestacional em $83 \%$ de diabéticas tipo l estudadas. Na nossa casuística, apesar das ITU e vulvovaginites estarem entre as complicações maternas mais freqüentes, ainda assim ocorreram em faixas inferiores a essa.

A HAS e a DHEG em gestantes com DM têm sido encontradas em uma freqüência 2 a 4 vezes superior à da população obstétrica normal, que está em torno de 7\% (13). No presente trabalho, a incidência dessas complicações foi menor nos 3 grupos, apesar do elevado percentual de obesidade nas pacientes com DM2 e DMG, fator que isoladamente relaciona-se a essas complicações.

Têm sido descritas, em gestações de diabéticas, taxas de mortalidade materna de mais de $50 \%$ (8). No nosso estudo não houve nenhum caso de óbito dentre as gestantes. A baixa freqüência de complicações micro e macroangiopáticas encontradas nas pacientes previamente diabéticas devem ter contribuído para isso.

Aborto é definido como óbito fetal que ocorre antes da 20a. semana de gestação (8). A incidência de aborto dentre diabéticas é controversa. De uma forma geral tem sido considerada como semelhante à da população geral, que está em torno de $10 \%$. Contudo, estudos prospectivos mais bem conduzidos têm demonstrado freqüências mais altas dessa complicação, entre 15 e $30 \%$ em gestantes com DMl sendo relacionados principalmente à hiperglicemia no período pré-concepção (16-18). Na nossa casuística, os abortos ocorreram, em DMl, em taxas inferiores a essas, apesar do mal controle metabólico $\left(\mathrm{HbA}_{1}: 11,1 \pm 2,9 \%\right)$ desse grupo no início do seguimento, sugerindo que outros fatores estejam também envolvidos na gênese dessa complicação.

Os partos por via abdominal são duas vezes mais comuns em mulheres com DMG que em gestantes sem DM (12). No nosso estudo, esses também foram os mais utilizados nos 3 grupos, com índices bastante elevados $(60,5$ a $79,5 \%$ dos partos). Estão relacionados, pelo menos em parte, ao grau de obesidade materna e macrossomia fetal nessas gestações, fatores que reconhecidamente interferem na decisão em relação ao tipo de parto.

A hipoglicemia neonatal, ou seja, a presença de glicemias menores que $35 \mathrm{mg} / \mathrm{dl}$ nas primeiras $12 \mathrm{hs}$ de vida, situa-se entre as complicações neonatais mais comuns em gestações de diabéticas, acometendo 10 a $50 \%$ delas. Os mecanismos envolvidos com a ocorrência dessa anormalidade não estão completamente definidos. Sabe-se, contudo, que a exposição crônica ao estado hiperglicêmico materno resulta em uma hiperplasia das ilhotas pancreáticas fetais com conseqüente hiperinsulinemia e hipoglicemia neonatal, teoria inicialmente proposta por Pedersen (13). Na nossa população, essa manifestação revelou-se a complicação neonatal mais freqüente, com mais de $50 \%$ dos recémnascidos acometidos nos 3 grupos.

Mesmo entre pacientes sem DM, os recémnascidos pré-termo são freqüentes, representando cerca de 7\% dos casos nos Estados Unidos. A prematuridade é considerada uma importante causa de morbi-mortalidade perinatal, sendo responsáveis por $75 \%$ dos casos complicados dentre os recém-nascidos naquela população. Embora indefinida a real incidência de partos pré-termo espontâneos em diabéticas, dada a elevada ocorrência de casos iatrogênicos, temse descrito taxas mais elevadas de até $31,1 \%(13)$. Na nossa amostra tivemos uma incidência elevada de prematuridade, especialmente no grupo com DMl $(53,7 \%)$.

Macrossomia é definida como um peso fetal ao nascimento acima do percentil 90 da população geral ou maior que $4 \mathrm{~kg}$. Ocorre em até $50 \%$ das pacientes com DMG e $40 \%$ em DMl, freqüência até 10 vezes superior à encontrada na população geral (9). Essa morbidade tem repercussões importantes, considerando que aumenta o risco de óbito fetal e outras complicações como tocotraumatismo, distocias, hipoglicemia 
neonatal, cardiomiopatia hipertrófica, malformações, trombose vascular, além de aumentar a indicação de partos cesáreas. Na nossa amostra essa alteração esteve entre as mais incidentes (tabela 4), no entanto, com uma freqüência menor que $30 \%$ nos 3 grupos.

Outra manifestação neonatal de ocorrência elevada em filhos de diabéticas é a icterícia. Essa alteração tem sido descrita em níveis de até $38 \%$ e $53 \%$, respectivamente, em recém-nascidos de gestantes com DMG e DMl $(9,19)$. Embora anormalidades como a policitemia e a prematuridade sejam também implicadas, o controle glicêmico materno parece o fator mais envolvido na sua patogênese. Na nossa casuística essa complicação foi também menos freqüente que outros estudos, tendo acometido predominantemente os recém-nascidos das gestantes com DMl, grupo que, como citado anteriormente, teve o pior controle glicêmico.

As malformações em recém-nascidos de diabéticas têm sido encontradas em freqüência 2 a 4 vezes superior à da população obstétrica normal, que está em torno de 1,7\% (20). Fatores genéticos, complicações crônicas do DM, como a microangiopatia e, principalmente, o controle metabólico materno inadequado, têm sido relacionados a esta complicação. No presente estudo, observamos freqüência também elevada dessa grave morbidade nas pacientes previamente diabéticas, que pode ser atribuído ao início tardio do seguimento dessas gestantes e à hiperglicemia no período inicial da gravidez.

Além do controle metabólico, alterações micro e macroangiopáticas têm sido também implicadas na morbi-mortalidade materna e fetal em diabéticas $(13,20)$. No presente estudo, observou-se uma baixa prevalência de complicações crônicas do DM nos grupos avaliados, o que pode ter contribuído para a freqüência de complicações materno-fetais observada no estudo. Essa baixa prevalência pode estar relacionada ao tempo de doença relativamente reduzido nos grupos com DM prévio, embora hajam algumas pacientes com mais de 5 anos de diagnóstico.

Várias complicações foram significativamente mais incidentes em recém-nascidos de gestantes com DMl. Podemos atribuir tal fato, pelo menos parcialmente, à elevada ocorrência de partos pré-termo nesse grupo, morbidade que reconhecidamente predispõe à ocorrência das demais.

Embora tenha havido melhora do controle metabólico nos grupos estudados, uma completa normalização dos níveis sangüíneos de glicose e hemoglobina glicada não foi atingida, o que provavelmente contribuiu para as taxas de complicações materno- fetais verificadas nas nossas pacientes. Uma abordagem efetiva quanto a aspectos importantes no manejo dessa condição, como o início do seguimento pré-natal, a detecção precoce e o diagnóstico correto de DMG, o controle metabólico durante a gestação e, nas pacientes previamente diabéticas, a programação da gravidez resultando em condições metabólicas adequadas para a concepção, talvez possam determinar futuramente uma menor morbi-mortalidade em gestações de diabéticas.

Comparando os resultados do presente estudo com os aqui realizados em períodos anteriores $(4,5)$, não foi observada redução das complicações ou melhora evidente do controle metabólico. Possivelmente, tal fato se deve à persistência do inicio tardio do acompanhamento pré-natal e da ocorrência de gestações não programadas em pacientes previamente diabéticas. Contudo, pôde-se observar um aumento do número de pacientes encaminhadas para seguimento no HCFMRP-USP, o que sugere maior atenção à gestante com DM na região de Ribeirão Preto nesse período. Além disso, houve também uma melhora na avaliação dessas pacientes e dos seus recém-nascidos, o que provavelmente possibilita a adoção futura de medidas mais efetivas.

\section{AGRADECIMENTOS}

Agradecemos à Sra. Nadia Bittar Garcia e ao Sr. Sebastião Lázaro Brandão Filho pelo auxílio técnico nas dosagens laboratoriais, e ao Dr. Rafael Kioshi Yano e à Nutricionista Valéria Laguna Salomão Ambrósio, cujas colaborações foram importantes para a realização desse trabalho.

\section{REFERÊNCIAS}

1. Kitzmiller JL, Cloherty JP, Younger MD, Tabatabaii A Rothchild SB, Sosenko I, et al. Diabetic pregnancy and perinatal morbidity. Am J Obstet Gynecol 1978; 131:560-80.

2. Widness JA, Cowett RM, Coustan DR, Carpenter MW, Oh W. Neonatal morbidities in infants of mothers with glucose intolerance in pregnancy. Diabetes 1985;34(Suppl 2):61-5.

3. Rudge MV, Calderon IM, Ramos MD, Abbade JF, Rugolo LM. Perinatal outcome of pregnancies complicated by diabetes and by maternal daily hyperglycemia not related to diabetes. A retrospective 10 years analysis. Gynecol Obstet Invest 2000;50:108-12.

4. Pimenta WP, Cunha SP, Foss MC. Gravidez e diabetes mellitus - experiência do período de 1980 a 1985 no Hospital das Clínicas da Faculdade de Medicina de Ribeirão Preto, Universidade de São Paulo. Rev Bras Ginecol Obstet 1988;3:54-61.

5. Paccola GMGF, Torquato MTG, Baima Filho J, Duarte G, Foss MC. Diabetes Mellitus e gravidez: acompan- 
hamento de 89 gestações de 1986 a 1991 no Hospital das Clínicas da Faculdade de Medicina de Ribeirão Preto - USP. Arq Bras Endocrinol Metab 1995;39(1):26-31.

6. National Diabetes Data Group. Classification and diagnosis of diabetes mellitus and other categories of glucose intolerance. Diabetes 1979;28:1039-57.

7. Metzger BE. Summary and recommendations of the Third International Workshop-Conference on Gestational Diabetes Mellitus. Diabetes 1991;40(Suppl 2):197-201.

8. Healy K, Jovanovic-Peterson L, Peterson CM. Pancreatic disorders of pregnancy. Pregestational diabetes. Endocrinol Metab Clin North Am 1995;24:73-101.

9. Landon MB, Gabbe SG. Diabetes mellitus. In: Barron WM, Lindheimer MD, eds. Medical Disorders During Pregnancy. $2^{\text {nd }}$ edition. Mosby-Year Book, 1995:63-88.

10. Goldman M, Kitzmiller JL, Abrams B, Cowan RM, Laros RK Jr. Obstetric complications with GDM. Effects of maternal weight. Diabetes 1991;40(Suppl 2):79-82.

11. Bevier WC, Jovanovic-Peterson L, Peterson CM. Pancreatic disorders of pregnancy. Diagnosis, management, and outcome of gestational diabetes. Endocrinol Metab Clin North Am 1995;24:103-38.

12. Casey BM, Lucas MJ, McIntire DD, Leveno KJ. Pregnancy outcomes in women with gestational diabetes compared with the general obstetric population. Obstet Gynecol 1997:90:869-73.

13. Rosen BM, Miodovnik M. Pregnancy and complications of type I diabetes: maternal and fetal implications. In LeRoith D, Taylor Sl, Olefsky JM, eds. Diabetes Mellitus. $1^{\text {s† }}$ ed, 1996;695-710.

14. Coustan DR, Reece EA, Sherwin RS, Rudolf MC, Bates SE, Sockin SM, et al. A randomized clinical trial of the insulin pump vs. intensive conventional therapy in diabetic pregnancies. JAMA 1986;255:631-6.

15. Stamler EF, Cruz ML, Mimouni F, Rosenn B, Siddiqi T, Khoury $\mathrm{J}$, et al. High infectious morbidity in pregnant women with insulin-dependent diabetes: an understated complication. Am J Obstet Gynecol 1990; 163:1217-21.

16. Miodovnik M, Skillman C, Holroyde JC, Butler JB, Wendel JS, Siddiai TA. Elevated maternal glycohemoglobin in early pregnancy and spontaneous abortion among insulin-dependent diabetic women. Am J Obstet Gynecol 1985; 153:439-42.

17. Miodovnik M, Mimouni F, Tsang RC, Ammar E, Kaplan L, Siddiqi TA. Glycemic control and spontaneous abortion in insulin-dependent diabetic women. Obstet Gynecol 1986;68:366-9.

18. Mills JL, Simpson JL, Driscoll SG, Jovanovic-Peterson L, Van Allen M, Aarons JH, et al. Incidence of spontaneous abortion among normal women and insulin-dependent diabetic women whose pregnancies were identified within 21 days of conception. N Engl J Med 1988; 319:1617-23.

19. Widness JA, Cowett RM, Coustan DR, Carpenter MW, On W. Neonatal morbidities in infants of mothers with glucose intolerance in pregnancy. Diabetes 1985;34 (Suppl 2):61-5.

20. Buchanan TA. Effects of maternal diabetes on intrauterine development. In: LeRoith D, Taylor SI, Olefsky JM, eds. Diabetes Mellitus. st $^{\dagger \dagger}$ ed, 1996;684-95.

\section{Endereço para correspondência:}

Milton Cesar Foss

Divisão de Endocrinologia e Metabologia

Departamento de Clínica Médica

Faculdade de Medicina de Ribeirão Preto-USP

Av. dos Bandeirantes, 3.900

14.048-900 Ribeirão Preto, SP

Fax: (016) 633-1144 